\title{
Uniqueness and least energy property for solutions to strongly competing systems ${ }^{\dagger}$
}

\author{
MONICA CONTI \\ Dipartimento di Matematica, Politecnico di Milano, \\ Piazza Leonardo da Vinci, 32, 20133 Milano, Italy \\ SUSANNA TERRACINI ${ }^{\S}$ \\ Dipartimento di Matematica e Applicazioni, Università degli Studi di Milano-Bicocca, \\ Via Bicocca degli Arcimboldi, 8, 20126 Milano, Italy \\ AND \\ GIANMARIA VERZINI ${ }^{\mathrm{II}}$ \\ Dipartimento di Matematica, Politecnico di Milano, \\ Piazza Leonardo da Vinci, 32, 20133 Milano, Italy
}

[Received 17 November 2005 and in revised form 14 June 2006]

For the reaction-diffusion system of three competing species:

$$
-\Delta u_{i}=-\mu u_{i} \sum_{j \neq i} u_{j}, \quad i=1,2,3,
$$

we prove uniqueness of the limiting configuration as $\mu \rightarrow \infty$ on a planar domain $\Omega$, with appropriate boundary conditions. Moreover we prove that the limiting configuration minimizes the energy associated to the system

$$
E(U)=\sum_{i=1}^{3} \int_{\Omega}\left|\nabla u_{i}(\mathbf{x})\right|^{2} \mathrm{~d} \mathbf{x}
$$

among all segregated states $\left(u_{i} \cdot u_{j}=0\right.$ a.e.) with the same boundary conditions.

\section{Introduction}

Spatial segregation may occur in population dynamics when two or more species interact in a highly competitive way. A wide literature is devoted to this topic, mainly for the case of competition models of Lotka-Volterra type (see e.g. [1, 7- -15, 17]). As a prototype for the study of this phenomenon, in [4] we consider the competition-diffusion system of $k$ differential equations:

$$
-\Delta u_{i}=-\mu u_{i} \sum_{j \neq i} u_{j}, \quad u_{i}>0 \quad \text { in } \Omega, \quad u_{i}=\varphi_{i} \quad \text { on } \partial \Omega, \quad i=1, \ldots, k .
$$

\footnotetext{
†Work partially supported by MIUR, Project "Metodi Variazionali ed Equazioni Differenziali Non Lineari”.

E-mail: monica.conti@polimi.it

§E-mail: suster@matapp.unimib.it

II E-mail: gianmaria.verzini@polimi.it
} 
Here $\Omega \subset \mathbb{R}^{N}$ is a bounded regular domain, and $\left(\varphi_{1}, \ldots, \varphi_{k}\right)$ is a given boundary datum (regular, nonnegative, and satisfying $\varphi_{i} \cdot \varphi_{j} \equiv 0$ for $i \neq j$ ). This system describes the stationary states of the evolution of $k$ species diffusing and competing for resources. The internal dynamics of the populations and the diffusion coefficients are trivialized (although a wide class of internal dynamics and diffusion coefficients could be considered, without providing substantial changes to the qualitative behaviour of the model; see [2, 3, 6]), while attention is focused on the coefficient $\mu$, the rate of mutual competition. As a matter of fact, it can be shown that large interaction induces the spatial segregation of the species in the limit configuration as $\mu \rightarrow \infty$. Precisely, the following result has been proved by the authors in [4]:

THEOREM 1.1 The system (1) admits a solution $\left(u_{1, \mu}, \ldots, u_{k, \mu}\right) \in\left(H^{1}(\Omega)\right)^{k}$ for every $\mu>0$. Moreover there exists $\left(\bar{u}_{1}, \ldots, \bar{u}_{k}\right) \in\left(H^{1}(\Omega)\right)^{k}$ such that $\bar{u}_{i} \cdot \bar{u}_{j}=0$ for $i \neq j$ and, up to subsequences,

$$
u_{i, \mu} \rightarrow \bar{u}_{i} \quad \text { in } H^{1} \text {, for every } i, \text { when } \mu \rightarrow \infty .
$$

Not only does the limiting configuration exhibit segregation, but also the differential structure of the model passes to the limit in the form of a system of distributional inequalities. We collect these properties by introducing the function class

$$
\mathcal{S}=\left\{U=\left(u_{1}, \ldots, u_{k}\right) \in\left(H^{1}(\Omega)\right)^{k}: \begin{array}{l} 
\\
u_{i} \geqslant 0, u_{i} \cdot u_{j}=0 \text { if } i \neq j, \text { in } \Omega \\
\\
-\Delta u_{i} \leqslant 0,-\Delta\left(u_{i}-\sum_{j \neq i} u_{j}\right) \geqslant 0
\end{array}\right\} .
$$

In fact, we have

$$
\left(\bar{u}_{1}, \ldots, \bar{u}_{k}\right) \in \mathcal{S} .
$$

Thus the study of $\mathcal{S}$ provides the understanding of the segregated states induced by strong competition. In this direction, a number of regularity properties, both of the densities and of the mutual interfaces, were obtained by the authors in [2,-6].

On the other hand, in [6] we studied the minimal energy configurations in the class of all possible segregated states. Precisely, let us define the energy of a $k$-tuple of densities as

$$
E(U)=\sum_{i=1}^{k} \int_{\Omega}\left|\nabla u_{i}(\mathbf{x})\right|^{2} \mathrm{~d} \mathbf{x} .
$$

In [6] we proved the following:

THEOREM 1.2 The problem

$$
\min \left\{E(U): u_{i} \in H^{1}(\Omega),\left.u_{i}\right|_{\partial \Omega}=\varphi_{i}, u_{i} \geqslant 0, u_{i} \cdot u_{j}=0 \text { if } i \neq j\right\}
$$

admits a solution. In addition

(a) the minimum is unique;

(b) the minimum belongs to $\mathcal{S}$;

(c) the minimum depends $H^{1}$-continuously on the boundary data endowed with the $H^{1 / 2}$ norm.

In particular, this result shows that the unique minimal energy configuration shares with the limiting states of system (1) the common property of belonging to $\mathcal{S}$. In the case of two populations, 
we can say much more: indeed, we know the explicit solution of both problems. Letting $\Phi$ be the harmonic extension of $\varphi_{1}-\varphi_{2}$ onto $\Omega$, it is easy to see that the pair $\left(\Phi^{+}, \Phi^{-}\right)$achieves the minimum (2), while in [4] we proved that it is the limit configuration of any sequence of pairs $\left(u_{1, \mu}, u_{2, \mu}\right)$ as $\mu \rightarrow \infty$. As a consequence, when $k=2$, the class $\mathcal{S}$ consists of exactly one element, the minimal one. One may wonder if this result can be extended to the case of three or more densities, a case in which no explicit solution is provided. Even without uniqueness, we have

Problem Is the minimal energy configuration the limiting state for the corresponding competitive system?

When $k \geqslant 3$, the answer is not obvious: it is worth noticing that while problem (2) has an evident variational structure, the reaction-diffusion system (1) is not variational at all (the nonlinear part is not of gradient type). Nevertheless, the present paper provides a partial positive answer to this question: indeed, we prove that, for three populations in the plane, the only element of $\mathcal{S}$ is the minimizer of the energy. Our main result is:

THEOREM 1.3 Let $k=3$, and $\Omega$ be a simply connected regular domain in $\mathbb{R}^{2}$. Then, for every admissible datum $\left(\varphi_{1}, \varphi_{2}, \varphi_{3}\right), \mathcal{S}$ consists of exactly one element.

This theorem, together with the results contained in [4, 6], immediately yields:

THEOREM 1.4 Let $\Omega$ be a simply connected regular domain in $\mathbb{R}^{2},\left(u_{1, \mu}, u_{2, \mu}, u_{3, \mu}\right)$ be any solution of (1) and $\left(\bar{u}_{1}, \bar{u}_{2}, \bar{u}_{3}\right)$ be the minimizer of (2). Then, for every $\alpha \in(0,1)$, the whole sequence $u_{i, \mu}$ tends to $\bar{u}_{i}$ in $H^{1} \cap C^{0, \alpha}$ as $\mu \rightarrow \infty$.

As we already observed, this is a remarkable fact, since it shows a deep connection between the variational problem (2) and the nonvariational system (1).

\section{Basic facts and notation}

Due to the conformal invariance of the problem, with no loss of generality we take

$$
\Omega=B=\left\{\mathbf{x} \in \mathbb{R}^{2}:|\mathbf{x}|<1\right\}
$$

(to be more precise, we assume $\partial \Omega$ to be of class $C^{1, \varepsilon}, \varepsilon>0$, so that also the Riemann map from $\Omega$ to $B$ is $C^{1, \varepsilon}$, see e.g. [16, Theorem 3.6, Chapter 3]). Throughout the paper we will assume that:

- $i, j, h$ denote integers between 1 and 3 .

- $\left(\varphi_{1}, \varphi_{2}, \varphi_{3}\right) \in\left(W^{1, \infty}(\partial B)\right)^{3}$ (an admissible boundary datum) is such that $\varphi_{i} \geqslant 0$ for every $i$, and $\varphi_{i} \cdot \varphi_{j}=0$ on $\partial B$ for $i \neq j$. The sets $\left\{\varphi_{i}>0\right\}$ are open connected arcs, and the function $\sum \varphi_{i}$ vanishes at exactly three points of $\partial B$ (the endpoints of the supports).

With the above notation, we define the class $\mathcal{S}$ of segregated densities as

$$
\mathcal{S}=\left\{U=\left(u_{1}, u_{2}, u_{3}\right) \in\left(H^{1}(B)\right)^{3}: \begin{array}{l}
u_{i} \geqslant 0, u_{i} \cdot u_{j}=0 \text { if } i \neq j, \text { in } B \\
-\Delta u_{i} \leqslant 0,-\Delta \widehat{u}_{i} \geqslant 0
\end{array}\right\},
$$

where the hat operator is defined on each component of a triple as

$$
\widehat{u}_{i}=u_{i}-\sum_{j \neq i} u_{j}
$$


In the following, with some abuse of notation, $U$ will denote both each triple $\left(u_{1}, u_{2}, u_{3}\right)$ in $\mathcal{S}$ and the function $\sum u_{i}$ in $H^{1}(B)$.

For any $U \in \mathcal{S}$ we define the sets (supports, or nodal regions)

$$
\omega_{i}=\left\{\mathbf{x} \in B: u_{i}(\mathbf{x})>0\right\} .
$$

The multiplicity of a point $\mathbf{x} \in \bar{B}$ (with respect to $U$ ) is

$$
m(\mathbf{x})=\sharp\left\{i: \text { measure }\left(\omega_{i} \cap B(\mathbf{x}, r)\right)>0 \forall r>0\right\} .
$$

The interfaces between two densities are defined as

$$
\Gamma_{i j}=\partial \omega_{i} \cap \partial \omega_{j} \cap\{\mathbf{x} \in B: m(\mathbf{x})=2\},
$$

so that $\omega_{i} \cup \omega_{j} \cup \Gamma_{i j}=B \backslash \bar{\omega}_{h}$. The supports $\omega_{i}$ and $\omega_{j}$ are said to be adjacent if $\Gamma_{i j}$ is not empty.

Below we list the principal properties of the elements of $\mathcal{S}$. We refer to [3, 6] for their proof, and for further details.

THEOREM 2.1 Let $U \in \mathcal{S}$.

(a) $U \in W^{1, \infty}(\bar{B})$. As a consequence, every $\omega_{h}$ is open and $\mathbf{x} \in \omega_{h}$ implies $m(\mathbf{x})=1$.

(b) $u_{i}$ is harmonic in $\omega_{i}, u_{i}-u_{j}$ is harmonic on $B \backslash \bar{\omega}_{h}$ (with $h \neq i, j$ ). In particular, if $\mathbf{x} \in \Gamma_{i j}$, then

$$
\lim _{\substack{\mathbf{y} \rightarrow \mathbf{x} \\ \mathbf{y} \in \omega_{i}}} \nabla u_{i}(\mathbf{y})=-\lim _{\substack{\mathbf{y} \rightarrow \mathbf{x} \\ \mathbf{y} \in \omega_{j}}} \nabla u_{j}(\mathbf{y}) \neq 0 .
$$

(c) For every $\mathbf{x} \in \bar{B}$ we have $1 \leqslant m(\mathbf{x}) \leqslant 3$, and $m(\mathbf{x})=3$ for a finite number of points.

(d) Each $\Gamma_{i j}$ is (either empty or) a connected arc, locally $C^{1}$, with endpoints either on $\partial B$ or at points with multiplicity three.

(e) If $m\left(\mathbf{x}_{0}\right)=3$, then $|\nabla U(\mathbf{x})| \rightarrow 0$ as $\mathbf{x} \rightarrow \mathbf{x}_{0}$. More precisely, we have the following asymptotic estimate:

$$
U(r, \vartheta)=C r^{3 / 2}\left|\cos \left(\frac{3}{2} \vartheta+\vartheta_{0}\right)\right|+o\left(r^{3 / 2}\right)
$$

(here $(r, \vartheta)$ denotes a system of polar coordinates around $\mathbf{x}_{0}$ ).

REMARK 2.1 Every $\omega_{i}$ is (pathwise) connected. Indeed, let $\omega_{i}=\alpha \cup \beta$, with $\alpha$ and $\beta$ disjoint, open, and nonempty. Recall that $u_{i}$ is continuous on $\bar{B}$, hence it vanishes (continuously) on $\partial \omega_{i} \backslash$ $\left\{\varphi_{i}>0\right\}$. Since $\left\{\varphi_{i}>0\right\}$ is connected, it cannot intersect both $\partial \alpha$ and $\partial \beta$ (recall that $u_{i}$ is strictly positive on this set). We infer that $u_{i}$ vanishes, for instance, on $\partial \beta$. But $u_{i} \in C(\bar{\beta})$, and it is harmonic on $\beta$. The classical maximum principle implies $u_{i} \equiv 0$ in $\beta$, a contradiction.

We recall that, by Theorem $1.2, \mathcal{S}$ has at least one element. In the next section we prove that it is unique.

\section{Uniqueness results}

To start with, we prove a topological result, stating that every triple in $\mathcal{S}$ has exactly one triple point.

Lemma 3.1 For every $U \in \mathcal{S}$ there exists exactly one point $\mathbf{a}_{U} \in \bar{B}$ such that $m\left(\mathbf{a}_{U}\right)=3$.

Proof. This is an easy consequence of the fact that, if $m(\mathbf{a})=3$, then any neighborhood of a contains points of every $\omega_{i}$, and hence every nonempty $\Gamma_{i j}$ satisfies $\bar{\Gamma}_{i j} \ni$ a. But every $\Gamma_{i j}$ is connected and starts from $\partial B$. 


\subsection{Uniqueness when the triple point is on the boundary}

The simplest situation is when $\mathbf{a}_{U}$ belongs to $\partial B$. In this case, one $\Gamma_{i j}$ is empty, and $\widehat{u}_{h}$ (for $h \neq i, j$ ) is harmonic on $B$.

Proposition 3.1 Let $U, V \in \mathcal{S}$ with $\mathbf{a}_{U} \in \partial B$. Then $V \equiv U$.

Proof. The assumption implies that $\mathbf{a}_{U}$ is the common endpoint of the supports of two data, say $\varphi_{1}$ and $\varphi_{3}$, and, as a consequence, $\Gamma_{13}$ is empty. Now, $\widehat{u}_{2}$ is $C(\bar{B})$ and, by Theorem 2.1 b), it is harmonic both on $B \backslash \bar{\omega}_{3}$ and on $B \backslash \bar{\omega}_{1}$. As $\bar{\omega}_{1} \cap \bar{\omega}_{3}=\left\{\mathbf{a}_{U}\right\}$ we deduce that $\widehat{u}_{2}$ is harmonic on $B$. We are going to prove that $\mathbf{a}_{V} \equiv \mathbf{a}_{U}$. This will conclude the proof: indeed, it will imply that also $\widehat{v}_{2}$ is harmonic, with the same boundary data, and thus $\widehat{u}_{2} \equiv \widehat{v}_{2}$; but they have exactly three nodal regions, therefore they correspond to the same triple in $\mathcal{S}$.

Assume by contradiction that $\mathbf{a}_{V} \neq \mathbf{a}_{U}$. Then $\mathbf{a}_{U}$ is a point of multiplicity 2 for $V$, belonging to the common boundary of $\left\{v_{1}>0\right\}$ and $\left\{v_{3}>0\right\}$. As a consequence, we can find a neighbourhood $\mathcal{N}$ of $\mathbf{a}_{U}$ such that $v_{2}$ vanishes on $\mathcal{N}$. On the other hand, by definition of multiplicity, $\left\{u_{2}>0\right\}$ intersects $\mathcal{N}$. We infer the existence of $\overline{\mathbf{x}} \in \mathcal{N}$ such that $u_{2}(\overline{\mathbf{x}})>0$ and $v_{1}(\overline{\mathbf{x}})+v_{3}(\overline{\mathbf{x}})>0$. Now,

$$
\left\{\begin{array} { l l } 
{ - \Delta \widehat { v } _ { 2 } \geqslant 0 } & { \text { in } B , } \\
{ \widehat { v } _ { 2 } = \widehat { \varphi } _ { 2 } } & { \text { on } \partial B , }
\end{array} \text { and } \quad \left\{\begin{array}{ll}
-\Delta \widehat{u}_{2}=0 & \text { in } B \\
\widehat{u}_{2}=\widehat{\varphi}_{2} & \text { on } \partial B
\end{array}\right.\right.
$$

It follows that $\widehat{v}_{2}-\widehat{u}_{2}$ is superharmonic on $B$ and (continuously) zero on $\partial B$, and so it is nonnegative in $B$. But

$$
\left(\widehat{v}_{2}-\widehat{u}_{2}\right)(\overline{\mathbf{x}})=-v_{1}(\overline{\mathbf{x}})-v_{3}(\overline{\mathbf{x}})-u_{2}(\overline{\mathbf{x}})<0
$$

a contradiction.

It remains to prove the uniqueness of the element when its triple point is in the interior of $B$. In this case we cannot proceed directly as in the previous arguments. We will start, providing a sort of local uniqueness.

\subsection{Interior triple point: local uniqueness}

Let $U \in \mathcal{S}$ be given, with trace $\left(\varphi_{1}, \varphi_{2}, \varphi_{3}\right)$ and triple point $\mathbf{a}=\mathbf{a}_{U}$. We want to prove a local dependence between the trace and the triple point. The key point is that if we know the triple point, we can construct a harmonic function closely related to $U$ : roughly speaking, the idea is to move the triple point of $U$ to the origin via a Möbius transformation, and then to double the angle in order to obtain an even number of nodal regions (compatible with an alternate sign rule). We introduce the transformation (using the complex notation: the reader will easily distinguish the index $i$ and the imaginary unit $l$, which appears, by the way, only in exponents)

$$
T_{\mathbf{a}}: \bar{B} \rightarrow \bar{B}, \quad T_{\mathbf{a}}(\mathbf{z})=\frac{\mathbf{z}+\mathbf{a}}{\overline{\mathbf{a}} \mathbf{z}+1}
$$

It is well known that $T_{\mathbf{a}}$ is a conformal map such that $T_{\mathbf{a}}(\partial B)=\partial B$ and $T_{\mathbf{a}}(\mathbf{0})=\mathbf{a}$. Also the map $\mathbf{z} \mapsto \mathbf{z}^{2}$ is conformal. We find that if $r=|\mathbf{z}|$ and $\vartheta=\arg \mathbf{z}$, the map $T_{\mathbf{a}}\left(\mathbf{z}^{2}\right)$ given in coordinates by

$$
\mathbf{z}=(r, \vartheta) \mapsto\left(r^{2}, 2 \vartheta\right) \mapsto\left(\Re \frac{r^{2} e^{2 \imath \vartheta}+\mathbf{a}}{\overline{\mathbf{a}} r^{2} e^{2 \imath \vartheta}+1}, \Im \frac{r^{2} e^{2 \imath \vartheta}+\mathbf{a}}{\overline{\mathbf{a}} r^{2} e^{2 \imath \vartheta}+1}\right)=\left(x_{1}, x_{2}\right)=\mathbf{x}
$$


is conformal. For every $\omega_{i}$, the set $\left\{\mathbf{z}: \mathbf{z}^{2} \in \omega_{i}\right\}$ has two open connected components, symmetric with respect to $\mathbf{0}$. We want to define a new harmonic function, having opposite signs on the two components, for every $i$. We se ${ }^{1}$

$$
w(\mathbf{z})= \begin{cases}\sigma(\mathbf{z}) u_{i}\left(T_{\mathbf{a}}\left(\mathbf{z}^{2}\right)\right) & \text { if } \mathbf{z} \in \bar{B}, T_{\mathbf{a}}\left(\mathbf{z}^{2}\right) \in \bar{\omega}_{i}, \\ \sum_{i=1}^{3} \sigma(\mathbf{z}) u_{i}\left(T_{\mathbf{a}}\left(\mathbf{z}^{2}\right)\right) & \text { if } \mathbf{z} \in \bar{B},\end{cases}
$$

where $\sigma$ is \pm 1 in such a way that $w$ has different signs on adjacent nodal regions. Then $w$ has six nodal regions, it is of class $C^{1}$ (by Theorem 2.1 b) and (e)) and

$$
w(-\mathbf{z})=-w(\mathbf{z})
$$

Theorem 2.1 also implies that $w$ is harmonic. We obtain

$$
\begin{cases}\Delta w=0 & \text { in } B \\ w=\gamma_{\mathbf{a}} & \text { on } \partial B\end{cases}
$$

where

$$
\gamma_{\mathbf{a}}(\mathbf{z})=\sum_{i=1}^{3} \sigma(\vartheta) \varphi_{i}\left(\frac{e^{2 \imath \vartheta}+\mathbf{a}}{\overline{\overline{\mathbf{a}}} e^{2 \imath \vartheta}+1}\right) .
$$

Clearly, also $\gamma_{\mathbf{a}}(-\mathbf{z})=-\gamma_{\mathbf{a}}(\mathbf{z})$. Observe that, given $\left(\varphi_{1}, \varphi_{2}, \varphi_{3}\right)$ and $\mathbf{a}$, (7) defines a unique $w$.

By standard calculation we obtain

$$
\begin{aligned}
\arg \left(\frac{e^{2 \imath \vartheta}+\mathbf{a}}{\overline{\mathbf{a}} e^{2 \imath \vartheta}+1}\right) & =\arg \left(\frac{e^{2 \imath \vartheta}+\mathbf{a}}{\overline{\mathbf{a}} e^{2 \imath \vartheta}+1} \cdot \frac{e^{-2 \imath \vartheta}\left(e^{2 \imath \vartheta}+\mathbf{a}\right)}{\mathbf{a} e^{-2 \imath \vartheta}+1}\right) \\
& =\arg \left(e^{-2 \imath \vartheta}\left(e^{2 \imath \vartheta}+\mathbf{a}\right)^{2}\right)=-2 \vartheta+2 \arg \left(e^{2 \imath \vartheta}+\mathbf{a}\right) .
\end{aligned}
$$

Thus, if we set

$$
\Theta_{\mathbf{a}}(\vartheta)=2 \arg \left(e^{2 \imath \vartheta}+\mathbf{a}\right)-2 \vartheta
$$

we can write, with the usual abuse of notation,

$$
\gamma_{\mathbf{a}}(\vartheta)=\sum_{i=1}^{3} \sigma(\vartheta) \varphi_{i}\left(\Theta_{\mathbf{a}}(\vartheta)\right)
$$

Remark 3.1 Let $U, V \in \mathcal{S}$ be such that $\mathbf{a}_{U}=\mathbf{a}_{V}$. Then $U \equiv V$. Indeed, two different triples with the same triple point should generate two different $w$ in (7) with the same boundary condition.

The above construction allows one to find whether or not a point in $B$ can be the triple point of a segregated state. We have

Lemma 3.2 Let $\left(\varphi_{1}, \varphi_{2}, \varphi_{3}\right)$ be an admissible boundary datum, and $\mathbf{a} \in B$. Then $\mathbf{a}$ is the triple point of an element of $\mathcal{S}$ (with datum $\varphi_{i}$ ) if and only if

$$
\nabla w(\mathbf{0})=\mathbf{0},
$$

where $w$ is defined by (7), (8).

\footnotetext{
1 We will keep writing $u_{i}(\mathbf{x})=u_{i}\left(r e^{\imath \vartheta}\right)=u_{i}(r, \vartheta), \varphi_{i}(\mathbf{x})=\varphi_{i}(\vartheta)$, and so on.
} 
Proof. If $\mathbf{a}=\mathbf{a}_{U}$ then $w$ satisfies (6). By conformality and Theorem 2.1) (e), we obtain $\nabla w(\mathbf{0})=\mathbf{0}$.

On the other hand, let $w$ be defined by (7) and let $\nabla w(\mathbf{0})=\mathbf{0}$. We can write the Fourier expansion of $\gamma_{\mathbf{a}}$,

$$
\gamma_{\mathbf{a}}(\vartheta)=\frac{A_{0}}{2}+\sum_{n=1}^{\infty}\left[A_{n} \cos n \vartheta+B_{n} \sin n \vartheta\right],
$$

and, since $\gamma_{\mathbf{a}}$ is odd (by (8)), we immediately obtain $A_{2 m}=B_{2 m}=0$. By standard separation of variables we infer $w=\sum_{m=0}^{\infty}\left[A_{2 m+1} \cos ((2 m+1) \vartheta)+B_{2 m+1} \sin ((2 m+1) \vartheta)\right] r^{2 m+1}$. Finally, by [11, we obtain $A_{1}=B_{1}=0$ and

$$
w(r, \vartheta)=\sum_{n=1}^{\infty}\left[A_{2 n+1} \cos ((2 n+1) \vartheta)+B_{2 n+1} \sin ((2 n+1) \vartheta)\right] r^{2 n+1} .
$$

Moreover,

$$
A_{3}^{2}+B_{3}^{2} \neq 0
$$

indeed, if not there would be $2 k$ arcs (where $k$ is the index of the first nonzero Fourier component), starting from $\mathbf{0}$, on which $w$ vanishes. Since a harmonic function does not admit closed level lines, this contradicts the fact that $\gamma_{\mathbf{a}}$ has exactly six zeroes (remember $(8)$, and the fact that $\left(\varphi_{1}, \varphi_{2}, \varphi_{3}\right)$ is an admissible datum). Now, $w$ is odd, so $|w|$ is even. Therefore we can invert the conformal map (5) on the half ball, obtaining a nonnegative function with exactly three nodal regions. It is now not difficult to prove that this function generates an element of $\mathcal{S}$, with datum $\left(\varphi_{i}\right)$ and triple point $\mathbf{a}$.

Now that we have characterized, for a given datum, the possible triple points, we can state the local dependence of these points on the data.

Proposition 3.2 Let $\left(\varphi_{1}, \varphi_{2}, \varphi_{3}\right)$ be an admissible boundary datum, and $\mathbf{a}_{\varphi} \in B$, so that (11) holds. Then there exist $\varepsilon, \delta>0$ such that, for every admissible datum $\left(\psi_{1}, \psi_{2}, \psi_{3}\right)$ with $\left\|\varphi_{i}-\psi_{i}\right\|_{W^{1, \infty}}<\varepsilon$ there exists exactly one $\mathbf{a} \psi$ satisfying (11) with datum $\left(\psi_{i}\right)$ and such that $\left|\mathbf{a}_{\psi}-\mathbf{a}_{\varphi}\right|<\delta$.

Proof. Without loss of generality (using the continuity of the fixed transformation $T_{\mathbf{a}_{\varphi}}$ ) we can assume that $\mathbf{a}_{\varphi} \equiv \mathbf{0}$.

We want to apply the implicit function theorem to the map

$$
\left(W^{1, \infty}(B)\right)^{3} \times B \rightarrow \mathbb{R}^{2}, \quad\left(\varphi_{1}, \varphi_{2}, \varphi_{3}, \mathbf{a}\right) \mapsto \nabla w(\mathbf{0}),
$$

in order to locally solve equation (11) for a (recall that the dependence of $w$ on $\left(\varphi_{1}, \varphi_{2}, \varphi_{3}\right)$ and $\mathbf{a}$ is given by (7), (8)). To this end, the only nontrivial thing to show is that

$$
\text { the } 2 \times 2 \text { jacobian matrix }\left.\quad \partial_{\left(a_{1}, a_{2}\right)} \nabla w(\mathbf{0})\right|_{\mathbf{a}=\mathbf{0}} \quad \text { is invertible. }
$$

Using the Poisson formula we can write

$$
w(\mathbf{x})=\frac{1-|\mathbf{x}|^{2}}{2 \pi} \int_{\partial B} \frac{\gamma_{\mathbf{a}}(\mathbf{y})}{|\mathbf{y}-\mathbf{x}|^{2}} \mathrm{~d}_{\mathbf{y}} s,
$$

which implies

$$
\nabla w(\mathbf{x})=-\frac{\mathbf{x}}{\pi} \int_{\partial B} \frac{\gamma_{\mathbf{a}}(\mathbf{y})}{|\mathbf{y}-\mathbf{x}|^{2}} \mathrm{~d}_{\mathbf{y}} s+\frac{1-|\mathbf{x}|^{2}}{\pi} \int_{\partial B} \frac{\mathbf{y}-\mathbf{x}}{|\mathbf{y}-\mathbf{x}|^{4}} \gamma_{\mathbf{a}}(\mathbf{y}) \mathrm{d}_{\mathbf{y}} s
$$


and

$$
\nabla w(\mathbf{0})=\frac{1}{\pi} \int_{\partial B} \mathbf{y} \gamma_{\mathbf{a}}(\mathbf{y}) \mathrm{d}_{\mathbf{y}} s .
$$

We choose the parametrization $\mathbf{y}=(\cos \vartheta, \sin \vartheta)$. Taking into account $(10)$ we obtain

$$
\nabla w(\mathbf{0})=\frac{1}{\pi}\left(\int_{0}^{2 \pi} \sum_{i=1}^{3} \sigma(\vartheta) \varphi_{i}\left(\Theta_{\mathbf{a}}(\vartheta)\right) \cos \vartheta \mathrm{d} \vartheta, \int_{0}^{2 \pi} \sum_{i=1}^{3} \sigma(\vartheta) \varphi_{i}\left(\Theta_{\mathbf{a}}(\vartheta)\right) \sin \vartheta \mathrm{d} \vartheta\right) .
$$

Now, differentiating (9) we infer that

$$
\left.\nabla_{\mathbf{a}} \Theta_{\mathbf{a}}(\vartheta)\right|_{\mathbf{a}=\mathbf{0}}=(-2 \sin 2 \vartheta, 2 \cos 2 \vartheta) .
$$

Since $\Theta_{\mathbf{0}}(\vartheta)=2 \vartheta$, we deduce that $\left.\partial_{\left(a_{1}, a_{2}\right)} \nabla w(\mathbf{0})\right|_{\mathbf{a}=\mathbf{0}}$ is equal to

$$
\frac{2}{\pi}\left(\begin{array}{l}
-\int_{0}^{2 \pi} \sum_{i=1}^{3} \sigma(\vartheta) \varphi_{i}^{\prime}(2 \vartheta) \sin 2 \vartheta \cos \vartheta \mathrm{d} \vartheta \int_{0}^{2 \pi} \sum_{i=1}^{3} \sigma(\vartheta) \varphi_{i}^{\prime}(2 \vartheta) \cos 2 \vartheta \cos \vartheta \mathrm{d} \vartheta \\
-\int_{0}^{2 \pi} \sum_{i=1}^{3} \sigma(\vartheta) \varphi_{i}^{\prime}(2 \vartheta) \sin 2 \vartheta \sin \vartheta \mathrm{d} \vartheta \int_{0}^{2 \pi} \sum_{i=1}^{3} \sigma(\vartheta) \varphi_{i}^{\prime}(2 \vartheta) \cos 2 \vartheta \sin \vartheta \mathrm{d} \vartheta
\end{array}\right)
$$

Let us compute (10) and the Fourier expansion in the proof of Lemma 3.2 when $\mathbf{a}=\mathbf{0}$. We have

$$
\sum_{i=1}^{3} \sigma(\vartheta) \varphi_{i}(2 \vartheta)=\gamma_{0}(\vartheta)=\sum_{n=1}^{\infty}\left[A_{2 n+1} \cos ((2 n+1) \vartheta)+B_{2 n+1} \sin ((2 n+1) \vartheta)\right],
$$

implies that

$$
\sum_{i=1}^{3} \sigma(\vartheta) \varphi_{i}^{\prime}(2 \vartheta)=\frac{1}{2} \sum_{n=1}^{\infty}(2 n+1)\left[-A_{2 n+1} \sin ((2 n+1) \vartheta)+B_{2 n+1} \cos ((2 n+1) \vartheta)\right] .
$$

This (together with the Werner formulas) allows us to compute the first term of 13):

$$
\begin{aligned}
& \int_{0}^{2 \pi} \sum_{i=1}^{3} \sigma(\vartheta) \varphi_{i}^{\prime}(2 \vartheta) \sin 2 \vartheta \cos \vartheta \mathrm{d} \vartheta \\
= & \frac{1}{4} \int_{0}^{2 \pi} \sum_{n=1}^{+\infty}(2 n+1)\left[-A_{2 n+1} \sin ((2 n+1) \vartheta)+B_{2 n+1} \cos ((2 n+1) \vartheta)\right][\sin 3 \vartheta+\sin \vartheta] \mathrm{d} \vartheta \\
= & \frac{1}{4} \int_{0}^{2 \pi}-3 A_{3} \sin ^{2} 3 \vartheta \mathrm{d} \vartheta=-\frac{3 \pi}{4} A_{3} .
\end{aligned}
$$

Analogous calculations prove

$$
\left.\partial_{\left(a_{1}, a_{2}\right)} \nabla w(\mathbf{0})\right|_{\mathbf{a}=\mathbf{0}}=\frac{3}{2}\left(\begin{array}{cc}
A_{3} & B_{3} \\
-B_{3} & A_{3}
\end{array}\right) .
$$

But we know (see (12) ) that $A_{3}^{2}+B_{3}^{2} \neq 0$. Therefore the jacobian matrix is invertible, concluding the proof. 


\subsection{Interior triple point: global uniqueness}

Proof of Theorem 1.3 By Theorem 1.2 we know that at least one element $M$ in $\mathcal{S}$ exists. Assume by contradiction that there exists another element $U \in \mathcal{S}$ with $U \neq M$ (that is, $U$ is not the minimal one). By Proposition 3.1 we have $\mathbf{a}_{U} \in B$. Again, without loss of generality (using the transformation $T_{\mathbf{a}_{U}}$ ) we can assume that $\mathbf{a}_{U} \equiv \mathbf{0}$. For $t>0$ we define

$$
U_{t}(\mathbf{x})=\frac{1}{t^{3 / 2}} U(t \mathbf{x})
$$

and we observe that

$$
U_{t} \in \mathcal{S}_{t}:=\left\{V=\left(v_{1}, v_{2}, v_{3}\right): \begin{array}{l}
v_{i} \geqslant 0, v_{i} \cdot v_{j}=0,-\Delta v_{i} \leqslant 0,-\Delta \widehat{v}_{i} \geqslant 0 \\
v_{i}(\mathbf{x})=\frac{1}{t^{3 / 2}} u_{i}(t \mathbf{x}) \text { for }|\mathbf{x}|=1
\end{array}\right\} .
$$

Now, $U_{t}$ has a limit as $t \rightarrow 0$. Indeed, by Theorem 2.1. (e) we can write

$$
U(r, \vartheta)=C r^{3 / 2}\left|\cos \left(\frac{3}{2} \vartheta+\vartheta_{0}\right)\right|+o\left(r^{3 / 2}\right) \quad \text { as } r \rightarrow 0,
$$

and hence

$$
U_{t}(r, \vartheta)=\frac{1}{t^{3 / 2}} U(t r, \vartheta)=C r^{3 / 2}\left|\cos \left(\frac{3}{2} \vartheta+\vartheta_{0}\right)\right|+\frac{o\left(t^{3 / 2} r^{3 / 2}\right)}{t^{3 / 2}}
$$

tends to

$$
U_{0}(r, \vartheta)=C r^{3 / 2}\left|\cos \left(\frac{3}{2} \vartheta+\vartheta_{0}\right)\right|
$$

Again $U_{0}$ belongs to

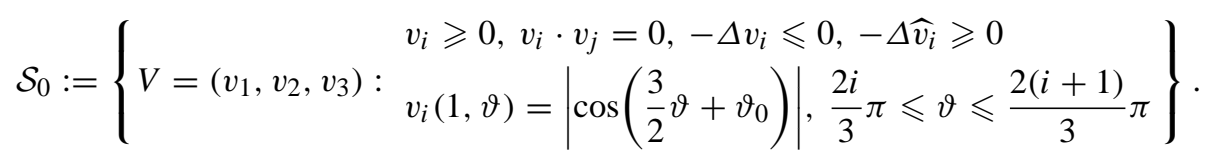

So we have a continuous path $U_{t}$ in $W^{1, \infty}(\bar{B})$ connecting $U$ and $U_{0}$. Denote by $M_{t}$ the minimum of $E$ in $\mathcal{S}_{t}$. While $U \not \equiv M$ by assumption, it is worth noticing that $U_{0} \equiv M_{0}$ is minimum. Indeed, the datum of $\mathcal{S}_{0}$ is symmetric and hence, by uniqueness of the minimal (Theorem 1.2 (a)), its triple point must be the origin; this implies (Remark 3.1) that $U_{0}$ is the minimal solution. Let

$$
\bar{t}=\sup \left\{t^{*} \geqslant 0: U_{t} \equiv M_{t} \text { for every } t \in\left[0, t^{*}\right]\right\} .
$$

By continuity of $E$ we immediately see that $U_{t^{*}} \equiv M_{t^{*}}$. On the other hand, we can find a sequence $\varepsilon_{n}>0$ such that

By Theorem 1.2(c), we have

$$
U_{t^{*}+\varepsilon_{n}} \not \equiv M_{t^{*}+\varepsilon_{n}}
$$

$$
M_{t^{*}+\varepsilon_{n}} \rightarrow M_{t^{*}} \text { a.e., which implies } \quad \mathbf{a}_{M_{t^{*}+\varepsilon_{n}}} \rightarrow \mathbf{a}_{M_{t^{*}}} .
$$

On the other hand, since $U_{t^{*}} \equiv M_{t^{*}}$, by construction we have

$$
U_{t^{*}+\varepsilon_{n}} \rightarrow M_{t^{*}} \text { in } W^{1, \infty}, \quad \text { which implies } \quad \mathbf{a}_{U_{t^{*}+\varepsilon_{n}}} \rightarrow \mathbf{a}_{M_{t^{*}}} .
$$

We infer that both $U_{t^{*}+\varepsilon_{n}}$ and $M_{t^{*}+\varepsilon_{n}}$ belong to $\mathcal{S}_{t^{*}+\varepsilon_{n}}$, and the distance between $\mathbf{a}_{U_{t^{*}+\varepsilon_{n}}}$ and $\mathbf{a}_{M_{t^{*}+\varepsilon_{n}}}$ is arbitrarily small. This contradicts Proposition 3.2, and concludes the proof. 


\section{REFERENCES}

1. Conti, M., \& FELli, V. Coexistence and segregation for strongly competing species in special domains. Preprint (2005).

2. Conti, M., Terracini, S., \& Verzini, G. Nehari's problem and competing species systems. Ann. Inst. H. Poincaré Anal. Non Linéaire 19 (2002), 871-888. [Zbl pre01881300| MR 1939088

3. Conti, M., Terracini, S., \& Verzini, G. An optimal partition problem related to nonlinear eigenvalues. J. Funct. Anal. 198 (2003), 160-196. Zbl pre01901767| MR 1962357

4. Conti, M., Terracini, S., \& Verzini, G. Asymptotic estimates for the spatial segregation of competitive systems. Adv. Math. 195 (2005), 524-560. Zbl pre02189415 $\mid$ MR 2146353

5. Conti, M., Terracini, S., \& Verzini, G. On a class of optimal partition problems related to the Fučík spectrum and to the monotonicity formulae. Calc. Var. Partial Differential Equations 22 (2005), 45-72. Zbl pre02135754 MR 2105968

6. Conti, M., Terracini, S., \& Verzini, G. A variational problem for the spatial segregation of reaction-diffusion systems. Indiana Univ. Math. J. 54 (2005), 779-815. Zbl pre02204353 MR 2151234

7. Cosner, C., \& C, A. Lazer. Stable coexistence states in the Volterra-Lotka competition model with diffusion. SIAM J. Appl. Math. 44 (1984), 1112-1132. Zbl 0562.92012 MR 0766192

8. Crooks, E. C. M., Dancer, E. N., Hilhorst, D., Mimura, M., \& Ninomiya, H. Spatial segregation limit of a competition-diffusion system with Dirichlet boundary conditions. Nonlinear Anal. Real World Appl. 5 (2004), 645-665. Zbl 1079.35008 MR 2079274

9. DANCER, E. N., \& DU, Y. H. Positive solutions for a three-species competition system with diffusion. I. General existence results. Nonlinear Anal. 24 (1995), 337-357. Zbl 0824.35033 MR 1312772

10. DANCER, E. N., \& DU, Y. H. Positive solutions for a three-species competition system with diffusion. II. The case of equal birth rates. Nonlinear Anal. 24 (1995), 359-373. Zbl 0824.35034 MR 1312773

11. DANCER, E. N., \& GUO, Z. M. Uniqueness and stability for solutions of competing species equations with large interactions. Comm. Appl. Nonlinear Anal. 1 (1994), 19-45. Zbl 0935.35074 MR 1280113

12. Dancer, E. N., Hilhorst, D., Mimura, M., \& Peletier, L. A. Spatial segregation limit of a competition-diffusion system. European J. Appl. Math. 10 (1999), 97-115. Zbl 0982.92031 MR 1687440

13. Eilbeck, J. C., Furter, J.E., \& López-Gómez, J. Coexistence in the competition model with diffusion. J. Differential Equations 107 (1994), 96-139. Zbl 0833.92010 MR 1260851

14. Lou, Y., MARTíneZ, S., \& NI, W. M. On $3 \times 3$ Lotka-Volterra competition systems with cross-diffusion. Discrete Contin. Dynam. Systems 6 (2000), 175-190. Zbl 1008.92035 MR 1739922

15. Lou, Y., \& NI, W. M. Diffusion, self-diffusion and cross-diffusion. J. Differential Equations 131 (1996), 79-131. Zbl 0867.35032 MR 1415047

16. Pommerenke, CH. Boundary Behaviour of Conformal Maps. Grundlehren Math. Wiss. 299, Springer, Berlin (1992). Zbl 0762.30001 MR 1217706

17. Shigesada, N., KaWASAKi, K., \& Teramoto, E. The effects of interference competition on stability, structure and invasion of a multispecies system. J. Math. Biol. 21 (1984), 97-113. Zbl 0568.92017 MR 0782629 